\title{
The role of proteomics in depression research
}

\author{
Daniel Martins-de-Souza $\cdot$ Laura W. Harris • \\ Paul C. Guest $\cdot$ Christoph W. Turck • \\ Sabine Bahn
}

Received: 6 November 2009/Accepted: 20 November 2009/Published online: 9 December 2009

(C) The Author(s) 2009. This article is published with open access at Springerlink.com

\begin{abstract}
Depression is a severe neuropsychiatric disorder affecting approximately $10 \%$ of the world population. Despite this, the molecular mechanisms underlying the disorder are still not understood. Novel technologies such as proteomic-based platforms are beginning to offer new insights into this devastating illness, beyond those provided by the standard targeted methodologies. Here, we will show the potential of proteome analyses as a tool to elucidate the pathophysiological mechanisms of depression as well as the discovery of potential diagnostic, therapeutic and disease course biomarkers.
\end{abstract}

Keywords Major depressive disorder (MDD) .

Unipolar MDD · Clinical MDD · Proteomics

\section{Depression}

Depression, unipolar depression, clinical depression or major depressive disorder (MDD) is a brain disorder which affects around $10 \%$ of world population [76]. It is characterized by low mood, low self-esteem, inappropriate guilt, thoughts of death and suicide, diminished

D. Martins-de-Souza and L. W. Harris contributed equally to this work.

D. Martins-de-Souza · C. W. Turck

Max Planck Institute for Psychiatry, Kraepelinstr. 2, 80804 Munich, Germany

e-mail:danms90@gmail.com

\section{W. Harris · P. C. Guest · S. Bahn $(\bowtie)$}

Department of Chemical Engineering and Biotechnology,

University of Cambridge, Tennis Court Road, Cambridge,

Cambridgeshire CB2 1QT, UK

e-mail:sb209@cam.ac.uk concentration, loss of interest or pleasure in normally enjoyable activities, and disturbance of sleep and appetite. In the United States, approximately $3 \%$ of depressed patients commit suicide and around $60 \%$ of people who commit suicide have MDD or another mood disorder (NIH). MDD is a leading cause of global disability and a risk factor for non-compliance with medical treatment [11].

MDD appears to be a multifactorial disease arising from genetic or metabolic predisposition in conjunction with environmental factors such as stressful life events and other stressors. Even though there is no anatomical hallmark, the risk of MDD may be due to significant variations in responsiveness of neural circuits and can be affected by other factors such as addiction or alterations in the dopaminergic system and the molecular mechanisms of memory [5].

Neuropathological studies have shown volume deficits, decreased neuronal size and glial pathology as well as decreased expression of synaptic markers, particularly in the anterior cingulate, orbitofrontal and dorsolateral prefrontal cortices [26]. Hippocampal volume reductions have been demonstrated by magnetic resonance imaging (MRI) [53] and may be linked to the effects of stress, hypercortisolaemia and hypothalamic-pituitary-adrenal (HPA) axis dysfunction, although evidence of cellular correlates for such effects is lacking [26]. Hippocampal neurogenesis has also been proposed as an underlying factor in the biology of MDD, mainly based on animal model studies and the effects of antidepressant medication [13]. For the last three decades, the main line of investigation into the neurobiology of MDD has been focused on changes in the monoamine neurotransmitters serotonin and norepinephrine, based on the known mechanisms of action of drugs with antidepressant activity. However, the relationship between low neurotransmitter levels and symptomatology is not simple and may be dependent on genetic background [1]. 
As only $60 \%$ of patients respond to current treatments and response time is lengthy in many cases with a high rate of relapse and treatment resistance [62], novel lines of research are warranted.

Numerous studies have used molecular profiling technologies to examine the brains of MDD patients with or without suicidality. Several groups have used microarray approaches to demonstrate abnormalities in the expression of transcripts related to synaptic transmission, particularly glutamate and GABAergic signalling, in the brains of depressed patients with or without suicide, especially in frontal and limbic regions $[8,34,64,65]$, with one negative study [68]. Subtle differences between suicides with MDD and non-MDD suicides have been detected leading some authors to postulate that there may be underlying differences in stress response [34]. Another study found two genes differentially expressed between suicide and nonsuicide patients across schizophrenia (SCZ) and bipolar disorder (BPD) subjects [33]. Further studies have used profiling methods to investigate the role of cholesterol in MDD, as altered blood cholesterol levels have been reported in MDD subjects, presenting a potential link with synaptic abnormalities in the brain. Lalovic et al. [38] profiled fatty acids in two regions of the frontal cortex and found no differences between suicide completers (with or without MDD) and controls, but found a decrease in cholesterol content in the frontal cortex of violent suicides only [39]. Further studies by the same group found differential expression of lipid metabolism and immune response genes using microarray analyses in frontal cortex of depressed suicide completers [40].

These significant and convergent findings are promising, and transcriptomics can provide information about a wide range of gene products and lead to the generation of new hypotheses. However, further investigation of the protein products of relevant genes is required in order to demonstrate a functional abnormality. In this respect, proteomics provides a useful complimentary approach and takes the understanding of brain function closer to a functional and, therefore, disease-determining endpoint. In recent years, proteomics has been used as a tool for better comprehension of human brain disorders as well as for the discovery of biomarkers for diagnosis, or for monitoring disease course, treatment response and the identification of novel therapeutic targets.

\section{Proteomics}

\section{Definition}

The term proteomics was originally defined as "the study of the total set of expressed proteins by a cell, tissue or organism at a given time under a determined condition" [75]. The proteome also includes the modifications made to a particular set of proteins produced by an organism or cellular system and this can vary with time and distinct requirements, stresses, or other environmental factors that a cell or organism is subjected to. Proteomic technologies allow the study of a subset of these proteins and differentially expressed proteins can usually be identified in comparisons of two or more different states.

Methods for proteome characterization

\section{$2 D-P A G E$}

Most proteomic analyses are performed using a combination of two-dimensional gel electrophoresis (2DE) for protein separation and mass spectrometry (MS) for protein identification. This combination of technologies allows the simultaneous separation of many hundreds of proteins in a single experiment. Direct comparison of 2DE profiles from different samples combined with unambiguous identification of the separated proteins by MS is the typical format followed by most researchers.

2DE has been extensively and successfully used in studies of brain tissue or body fluids of psychiatric disorders such as SCZ [46, 49, 60] and BPD [2, 17] as well as in studies of neurodegenerative disorders such as Alzheimer's disease (AD) $[25,72,74]$. These studies have identified a number of proteins that could be involved in disease pathogenesis, increasing the understanding of such disorders. Despite the separation power of this approach, 2DEMS-based proteomics presents some limitations such as a difficulty in detecting low-abundance, acidic or basic proteins as well as proteins with extremes of high or low molecular weight [24]. This has led researchers to find alternative ways to study the proteome.

\section{Shotgun proteomics}

An alternative direct MS-based approach has been developed to avoid such drawbacks [44]. This is generally known as shotgun proteomics or liquid chromatographytandem mass spectrometry (LC-MS/MS) which uses a combination of chromatographic steps prior to MS analyses in a high-throughput way. Protein quantitation of shotgun approaches, especially when stable-isotope methods are used, is more accurate than the 2DE-MS approach. One potential drawback of shotgun approaches is that they are not capable of providing direct information on intact proteins, as is the case for 2DE. However, shotgun proteomics has been used in SCZ studies, revealing some differentially expressed proteins that have not be found by $2 \mathrm{DE}$ methods $[47,48]$. 


\section{Validation experiments}

While 2DE and shotgun approaches are used to reveal the global protein expression of a given tissue, other methods such as Western blot (WB), enzyme-linked immunoadsorbent assay (ELISA), multiple reaction monitoring (MRM) mass spectrometry and antibody arrays are employed commonly for validation of differentially expressed proteins. Validation is necessary to demonstrate that the differences found in a limited small set of samples can also be found in a broader universe of samples, considering factors such as age, gender and different ethnicities. Moreover, there is a necessity to implement and improve high-throughput methods for validation studies such that high sample numbers are not a limiting factor.

\section{Luminex bead-based approach}

The recent introduction of fluorescent bead-based technologies allows the simultaneous measurement of multiple analytes in individual small-volume samples, revolutionizing proteomic analyses. This analytical platform is also suitable for further development of convenient, rapid, sensitive and specific diagnostic assays. This technology employs multiplexed dye-coded microspheres, coated with specific capture reagents for assaying targeted analytes within clinical samples. Identification and quantitation of the analytes are achieved by laser excitement within an analyser. The format allows multiplexing of up to 100 distinct assays for a single sample. This approach minimizes sampling errors, the amount of sample required for each analysis and the requirements and costs of assay reagents. Such technology, still not well implemented in studies of brain disorders, has already been applied successfully in numerous clinical studies or biomarker discovery projects of diseases such as epithelial ovarian cancer [6], scleroderma [15], coronary artery disease [23], myocardial infarction [18], autoimmune disorders [10] and sickle cell anaemia [42].

\section{Metabolomics}

Comparative proteome analyses of human brain tissue have led to the identification of biochemical pathways and consequently have provided some indication of the associated metabolites. The quantitation of metabolites using diverse approaches can provide a more complete picture of brain activities which may be useful for comprehension of the biochemical processes as well as a means of providing a source of non-protein-based biomarkers.

Targeted analysis of metabolites has been used to functionally validate protein expression changes. Since we found that key glycolysis enzymes were differentially expressed in
SCZ thalamus, we measured the levels of pyruvate and NADPH using a simple enzymatic assay and confirmed that these metabolites were also differentially expressed [51]. The advent of high-resolution proton nuclear magnetic resonance spectroscopy $\left({ }^{1} \mathrm{H}-\mathrm{NMR}\right)$ has provided a means of multiplexing analyses of metabolites providing greater accuracy and higher throughput. ${ }^{1} \mathrm{H}-\mathrm{NMR}$ metabolomics has been used to observe the effects of a number of psychotropic drugs on rat brain metabolites and significant differences were found regarding $N$-acetylaspartate (NAA) levels [54]. Moreover, high-performance liquid chromatography (HPLC) has been used to quantify significant differences in the polyamines putrescine and spermidine in high anxiety-related behaviour (HAB) and low anxietyrelated behaviour (LAB) brain tissue extracts [12].

Since all of the molecular profiling methods described here have their strengths and weaknesses, the combined use of two or more of these would be best to maximize coverage of the relevant molecular pathways such as those described by Salim et al. [63] in the characterization of proteome differences that occur during the differentiation of neural precursor cells or as applied in the proteome analysis of SCZ thalamus compared to healthy controls by Martins-de-Souza et al. [51].

\section{Proteomics in psychiatric disorders}

Despite considerable efforts, there is still a lack of animal models for psychiatric disorders, with only a few that are well-established. For SCZ, e.g., it is difficult to model symptoms in animals that reflect the human condition. This is one of the main driving reasons why researchers have focused mainly on the analysis of human postmortem brain tissues and fluids from SCZ and BPD patients. The main goal of these human studies is a better comprehension of the pathophysiology of such disorders as well as identification of biomarker candidates and potential novel targets. Indeed, such proteome analyses have successfully identified components of molecular pathways involved in psychiatric disorders, thereby demonstrating the potential of these methods in this field of research.

\section{Schizophrenia}

Nine different brain regions from SCZ patients have been subjected to proteomic analyses using different methodologies $[2,3,9,17,31,46-49,51,59,60,69]$. In addition, serum and cerebrospinal fluid (CSF) from SCZ patients have been analysed [30, 43, 51, 61]. The most frequent protein alterations identified were related to energy, oligodendrocyte and phospholipid metabolism as well as $\mathrm{Ca}^{2+}$ homeostasis [52]. 
In SCZ brain tissue, dysregulation of pathways associated with reactive oxygen species has been observed [77] as well as alterations in mitochondrial oxidative phosphorylation [27, 73] and glucose metabolism [4]. Proteome analyses have identified differential expression of proteins involved in those processes, such as key enzymes associated with glucose metabolism including aldolase $\mathrm{C}$ (ALDOC), gamma enolase (ENO2), aconitase (ACO2), hexokinase (HK1), glyceraldehyde-3-phosphate dehydrogenase (GAPDH), a number of subunits of mitochondrial ATPase and proteins associated to oxidative stress [52].

Oligodendrocytes are responsible for myelination of axons in the central nervous system (CNS). Reduction or malformation of the myelin sheath can result in leakage and reduced propagation of nerve impulses [14]. Dysfunction of oligodendrocytes has been demonstrated for SCZ using imaging techniques such as diffusion tensor imaging (DTI) and magnetization transfer imaging (MTI) $[20,36]$. In addition, proteome analyses have confirmed this through identification of differentially expressed proteins such as Myelin basic protein (MBP), myelin oligodendrocyte glycoprotein (MOG) and $2^{\prime}, 3^{\prime}$-cyclic nucleotide, $3^{\prime}$-phosphodiesterase (CNP) [52].

Potential biomarkers such as apolipoprotein A1 (APOA1) have been identified in CSF, red blood cells and serum from first-onset drug-naive SCZ patients [30, 43, 61]. In addition, apolipoprotein $\mathrm{A} 2$ and $\mathrm{A} 4$, and transferrin have been identified as differentially expressed in serum of SCZ subjects [43].

\section{Bipolar disorder}

Proteome analyses of BPD human brain tissue have also been performed. Dihydropyrimidinase-related protein-2 (DPYSL2) and glial fibrillary acid protein (GFAP) were found to be decreased in the frontal cortex (FC-BA10) suggestive of effects on brain development. The differential expression of several tubulin subunits suggested that cytoskeletal dysfunction may be an important component of BPD through an analysis of the anterior cingulate cortex (ACC-BA24) [2].

One common finding in proteomic studies of distinct brain regions from BPD patients is suggestive of a dysfunction in energy metabolism [2, 31, 58], more prominently in the dorsolateral prefrontal cortex (DLPFC-BA9) where half of the identified differentially expressed proteins were involved in these pathways [59].

Animal models

The study of animal models of psychiatric disorders has advantages over analyses of the corresponding human tissue, since laboratory animals show greater genetic homogeneity and are not fraught with confounding factors such as alcohol and tobacco consumption. However, the direct study of human samples has a key advantage over animal models in that the generated data are related to the disease itself, and in many cases the validity of psychiatric animal models has not been fully determined.

\section{Proteomics in depression research}

Proteome of human samples

At present, only two brain regions, the frontal cortex (FC) and accumbens (ACC), from depressed patients have been subjected to proteomic analyses, revealing differentially expressed proteins [2, 31]. Altered expression of DPYSL2 was common to both studies, although this was downregulated in FC and upregulated in ACC. DPYSL2 plays a role in nervous system development and cell differentiation by regulating axonal guidance, neuronal growth cone collapse and cell migration, suggestive of alterations in brain development in depressed patients. The differential expression of carbonic anhydrase (CA2) and ALDOC was also common to both studies, implicating effects on energy metabolism.

Both of these studies used brain tissue samples from the Stanley Neuropathology Consortium and analysed samples from MDD, SCZ, BPD and control subjects simultaneously. In addition, studies using CSF from depressed patients have been carried out, but only in a validation context for SCZ findings [29]. Thus far, no systematic proteomic study has been carried out on brain tissues from depressed patients. Considering the importance of this disorder and the effects on society, more studies in human tissue and fluids from such patients are required.

Proteome studies of depression using animal models

The difficulty in comprehending the complex regulation of gene and protein expression and interactions with environmental and external factors such as drug treatment is the main reason that researchers have focused their efforts on investigating samples from animal models rather than those from the human disease condition.

Most of the proteomics studies relating to MDD have focused on the response to drug treatments. Nowadays, MDD treatment is widely based on the prescription of biogenic-amine-based agents that selectively block the uptake of serotonin and/or norepinephrine. Such treatments differ from previous long-standing treatments, based on the increase of serotonin and norepinephrine levels in synapses, even though the therapeutic drawbacks still remain which are most likely intrinsic to treatment mechanism 
[70]. It is known that the demonstrated reduction of hippocampal volume in depressed patients [66] might be affected by antidepressants which reverse the shrinkage of hippocampal neurons, improving the symptoms of the disease [16]. Some articles have also demonstrated that antidepressant treatment may modulate molecules associated with neurogenesis [45] such as brain-derived neurotrophic factor (BDNF) [57]. This has been demonstrated in brain tissue from knockout mice for neurokinin-1 (NK1) receptor gene, since antagonists for this receptor show antidepressant activity [55]. Interestingly, BDNF can induce antidepressant effects [67] probably because this molecule can increase neurogenesis in hippocampus [41] as well as 5HT and noradrenaline [21,37]. Despite all the knowledge regarding treatment, the mechanisms of antidepressant drugs still require further elucidation. Proteome analysis could be helpful for the better understanding of antidepressant treatments, especially because proteome analyses can reveal the proteins involved in such mechanisms as well as the identification of potential biomarkers [50].

Impairments in the HPA axis have led most proteomic researchers to investigate the hypothalamus in these models. The known imbalances in monoamine levels in brains from depressed patients have driven comparative proteomic studies on the mechanism of action of monoamine reuptake inhibitors such as venlafaxine, a serotonin-norepinephrine reuptake inhibitor (SNRI), or fluoxetine, a selective serotonin reuptake inhibitor (SSRI). Adult rats treated for 2 weeks with such drugs have shown the differential expression of more than 30 proteins that play roles in neurogenesis, maintenance of neuronal processes and regeneration [32]. Studies of mice treated with chronic corticosterone have been carried out in hypothalamus, hippocampus and cerebral cortex, resulting in identification of alterations in energy pathway enzymes and phenylalanine, glutamate and nitrogen metabolism. Moreover, pathway analysis suggested an alteration in cell morphology in the hippocampus and cerebral cortex, and cell death/ survival pathways in the hypothalamus of these mice [71]. Other proteomic studies have used a mixture of neural and glial cells in culture after exposure to $1 \mathrm{mM}$ of paroxetine for 14 days in order to understand antidepressant action. The observed upregulation of sepiapterin reductase (SPR) was interesting as this enzyme is involved in modulating the synthesis of neurotransmitters such as serotonin. Moreover, the upregulation of heat shock protein 9A and protein disulfide isomerase as well as the downregulation of creatine kinase, prohibitin, glial fibrillary acidic protein and vimentin suggests that these may be involved in the mechanism of action of antidepressant medication and may also offer some insight regarding the pathogenesis of MDD.
The effects of fluoxetine in guinea pigs showed alterations in the proteome regarding neurofilaments, suggestive of effects on synaptic remodelling. Validation studies using immunohistochemistry and electron microscopy were carried out showing an increase in post-synaptic neurons with split post-synaptic densities, which may explain why antidepressants take weeks to months before their therapeutic effects are realized [22].

Proteomic studies of hippocampus from a chronic stress rat model of MDD identified 27 differentially expressed proteins with a role in neurogenesis and oxidative metabolism [56]. Another study reported on analysis of the hippocampal proteome of different treatment groups using the chronic mild stress model of MDD in rats with the aim of identifying potential biomarkers for anhedonia, escitalopram resistance and stress resilience. Thirteen proteins were identified as differentially expressed between the groups and interestingly, DPYSL2 was identified as a biomarker for escitalopram resistance. Moreover, the authors claimed that cellular plasticity is an important issue for the understanding of the molecular mechanism of MDD [7].

Extensive proteomic studies have been conducted regarding the $\mathrm{HAB}$ and $\mathrm{LAB}$ mice model. $\mathrm{HAB}$ mice are hyperanxious representing a comorbid depression-like behaviour according to elevated plus-maze (EPM), tail suspension (TST) and forced swim tests (FST) [28]. Proteome analyses of $\mathrm{HAB} / \mathrm{LAB}$ mice led to the identification of glyoxalase-I as a protein marker downregulated in multiple brain areas [35] and an alternate enolase phosphatase isoform in HAB mice [12].

\section{Limitations}

Despite the contribution of studies of animal models, investigation of the human brain is essential to further understand the causes and symptomatology of MDD, and particularly in the investigation of suicidality. Nonetheless, careful consideration of experimental design and potential confounders must be taken. This is as much a problem for proteomic investigations as with other areas of research. As with all psychiatric disorders, some awareness of the role of lifestyle factors is important. Chronically ill patients may have different diets, access to healthcare and exercise levels compared to control subjects. Given the known effects of exercise on hippocampal function, records of exercise levels, body mass indices (BMIs) and measurements of metabolic parameters may be necessary in the interpretation of postmortem findings. Furthermore, as medication is generally the first-line approach for treatment of MDD, the majority of study subjects will have been medicated at some stage of the illness. This can only be addressed by collecting samples from subjects at first presentation for peripheral studies, and in postmortem 
studies by careful analysis of patient data and comparison with the known effects of antidepressant medication. Alcohol and substance misuse are common problems amongst MDD patients and represent important confounds as the neurobiological effects of these drugs may overlap with those found in non-abusing MDD patients. Indeed, whether MDD arises as cause or effect of substance misuse merits contemplation. Similarly, there is a high degree of co-morbidity with MDD, suicide and other disorders such as $\mathrm{AD}, \mathrm{BD}$ and SCZ. Moreover, the study of mood disorders requires consideration of the state versus trait problem, namely whether the detected changes are simply correlates of mood or a reflection of the underlying biology which predisposes the subject to such abnormal mood states.

Attempts to assess and control for these factors can be made by studying a range of patient types and subtypes postmortem, and by comparison with data from living patients and preclinical models. Bioinformatic strategies can also be used to distinguish signal from noise and incorporate relevant variables in the data analysis pipeline. Proteomics presents an ideal route to address these factors as the methods are applicable across species and in all tissues and fluids of the human body. However, some confounds will inevitably remain and this must be taken into account when considering the data.

\section{Conclusions and perspectives}

The sequencing of human genome brought in the 1990s a renewed hope regarding the molecular understanding and more effective treatments for many diseases such as psychiatric disorders. But the exceedingly large and valuable amount of data that the human genome project provided requires considerable further study and data mining to maximize the impact. Proteomic studies may offer further insights, helping to unlock the function of the genome. Proteome generated data can be interpreted through systems biology analysis in order to comprehend the causes and consequences of complex psychiatric disorders such as MDD. Moreover, identification of differentially expressed proteins using proteomics, principally in body fluids, can provide biomarkers to facilitate the development of early diagnostics, more effective treatments and other ends.

Most studies regarding protein expression in MDD have been carried out in animal models as described above, since there is a necessity to eliminate environmental and external factors that can confound the findings as well as to observe the effects of treatments. Despite these efforts, there is considerable scope for understanding more about this disorder, which could lead to identification of diagnostic/prognostic biomarkers and potential novel therapeutics. For example, it is known that physical exercises such as running can reverse hippocampal dysfunction in depressed patients, and this could be mediated by proteins such as the pituitary nerve growth factor VGF [19]. Proteomic analysis of models involving over- or underexpression of VGF would help to compose a better overview of neurotrophic signalling pathways in MDD. Moreover, despite the importance of studying animal models, it is also necessary to study human tissues such as postmortem brain tissue and peripheral fluids such as blood and CSF from human patients in order to construct a more complete scenario of MDD, as has been done in SCZ and BPD studies. For example, studying body fluids from depressed patients who have attempted suicide could lead to identification of markers for suicide risk as well as biomarkers of brain function. These studies could allow a ranking for risk of suicidal behaviour according to a proteomic fingerprint.

Even with all of the invested efforts to date, the field of proteomics in MDD studies still needs to be explored more deeply.

Acknowledgments We would like to thank the Stanley Medical Research Institute and all tissue donors and their families for comprehending how important their consent is to our research and to the lives of patients.

Open Access This article is distributed under the terms of the Creative Commons Attribution Noncommercial License which permits any noncommercial use, distribution, and reproduction in any medium, provided the original author(s) and source are credited.

\section{References}

1. Aan Het Rot M, Mathew SJ, Charney DS (2009) Neurobiological mechanisms in major depressive disorder. CMAJ 180(3):305-313

2. Beasley CL, Pennington K, Behan A, Wait R, Dunn MJ, Cotter D (2006) Proteomic analysis of the anterior cingulate cortex in the major psychiatric disorders: evidence for disease-associated changes. Proteomics 6:3414-3425

3. Behan A, Byrne C, Dunn MJ, Cagney G, Cotter DR (2009) Proteomic analysis of membrane microdomain-associated proteins in the dorsolateral prefrontal cortex in schizophrenia and bipolar disorder reveals alterations in LAMP, STXBP1 and BASP1 protein expression. Mol Psychiatry 14:601-613

4. Ben-Shachar D (2002) Mitochondrial dysfunction in schizophrenia: a possible linkage to dopamine. J Neurochem 83:12411251

5. Berke JD, Hyman SE (2000) Addiction, dopamine, and the molecular mechanisms of memory. Neuron 25(3):515-532 (review)

6. Bertenshaw GP, Yip P, Seshaiah P, Zhao J, Chen TH et al (2008) Multianalyte profiling of serum antigens and autoimmune and infectious disease molecules to identify biomarkers dysregulated in epithelial ovarian cancer. Cancer Epidemiol Biomarkers Prev 17(10):2872-2881

7. Bisgaard CF, Jayatissa MN, Enghild JJ, Sanchéz C, Artemychyn R, Wiborg O (2007) Proteomic investigation of the ventral rat hippocampus links DRP-2 to escitalopram treatment resistance 
and SNAP to stress resilience in the chronic mild stress model of depression. J Mol Neurosci 32(2):132-144

8. Choudary PV, Molnar M, Evans SJ, Tomita H, Li JZ, Vawter MP et al (2005) Altered cortical glutamatergic and GABAergic signal transmission with glial involvement in depression. Proc Natl Acad Sci USA 102:15653-15658

9. Clark D, Dedova I, Cordwell S, Matsumoto I (2006) A proteome analysis of the anterior cingulate cortex gray matter in schizophrenia. Mol Psychiatry 11:459-470

10. Delaleu N, Immervoll H, Cornelius J, Jonsson R (2008) Biomarker profiles in serum and saliva of experimental Sjogren's syndrome: associations with specific autoimmune manifestations. Arthritis Res Ther 10(1):R22

11. DiMatteo MR, Lepper HS, Croghan TW (2000) Depression is a risk factor for noncompliance with medical treatment: metaanalysis of the effects of anxiety and depression on patient adherence. Arch Intern Med 160:2101-2107

12. Ditzen C, Varadarajulu J, Czibere L, Gonik M, Targosz BS et al (2009) Proteomic-based genotyping in a mouse model of trait anxiety exposes disease-relevant pathways. Mol Psychiatry [Epub ahead of print]

13. Drew MR, Hen R (2007) Adult hippocampal neurogenesis as target for the treatment of depression. CNS Neurol Disord Drug Targets 6:205-218

14. Du Y, Dreyfus CF (2006) Oligodendrocytes as providers of growth factors. J Neurosci Res 68:647-654

15. Duan H, Fleming J, Pritchard DK, Amon LM, Xue J et al (2008) Combined analysis of monocyte and lymphocyte messenger RNA expression with serum protein profiles in patients with scleroderma. Arthritis Rheum 58(5):1465-1474

16. Duman RS, Malberg J, Nakagawa S, D'Sa C (2000) Neuronal plasticity and survival in mood disorders. Biol Psychiatry 48:732-739

17. English JA, Dicker P, Föcking M, Dunn MJ, Cotter DR (2009) 2D DIGE analysis implicates cytoskeletal abnormalities in psychiatric disease. Proteomics 9:3368-3382

18. Escobar GP, Lindsey ML (2007) Multi-analyte profiling of postmyocardial infarction plasma samples. FASEB J 21:746.11

19. Fabel K, Fabel K, Tam B, Kaufer D, Baiker A et al (2003) VEGF is necessary for exercise-induced adult hippocampal neurogenesis. Eur J Neurosci 18(10):2803-2812

20. Foong J, Maier M, Barker GJ, Brocklehurst S, Miller DH, Ron MA (2000) In vivo investigation of white matter pathology in schizophrenia with magnetization transfer imaging. J Neurol Neurosurg Psychiatry 68:70-74

21. Gould E (1999) Serotonin and hippocampal neurogenesis. Neuropsychopharmacology 21:46S-51S

22. Guest PC, Knowles MR, Molon-Noblot S, Salim K, Smith D et al (2004) Mechanisms of action of the antidepressants fluoxetine and the substance $\mathrm{P}$ antagonist L-000760735 are associated with altered neurofilaments and synaptic remodeling. Brain Res 1002(1-2):1-10

23. Gurbel PA, Kreutz RP, Bliden KP, DiChiara J, Tantry US (2008) Biomarker analysis by fluorokine multianalyte profiling distinguishes patients requiring intervention from patients with longterm quiescent coronary artery disease: a potential approach to identify atherosclerotic disease progression. Am Heart $\mathbf{J}$ 155(1):56-61

24. Gygi SP, Corthals GL, Zhang Y, Rochon Y, Aebersold R (2000) Evaluation of two-dimensional gel electrophoresis-based proteome analysis technology. Proc Natl Acad Sci USA 97(17):93909395

25. Harris LJW, Swatton JE, Wengenroth M, Wayland M, Lockstone HE, Holland A, Faull RLM, Lilley KS, Bahn S (2007) Differences in protein profiles in schizophrenia prefrontal cortex compared to other major brain disorders. Clin Schizophrenia Relat Psychoses 1:21-39

26. Harrison PJ (2002) The neuropathology of primary mood disorder. Brain 125(Pt 7):1428-1449

27. Hazlett EA, Buchsbaum MS, Byne W, Wei TC, Spiegel-Cohen J et al (1999) Three-dimensional analysis with MRI and PET of the size, shape, and function of the thalamus in the schizophrenia spectrum. Am J Psychiatry 156:1190-1199

28. Henniger MS, Ohl F, Holter SM, Weissenbacher P, Toschi N et al (2000) Unconditioned anxiety and social behaviour in two rat lines selectively bred for high and low anxiety-related behaviour. Behav Brain Res 111:153-163

29. Huang JT, Leweke FM, Oxley D, Wang L, Harris N et al (2006) Disease biomarkers in cerebrospinal fluid of patients with firstonset psychosis. PLoS Med 3(11):e428

30. Huang JT, Wang L, Prabakaran S, Wengenroth M, Lockstone HE et al (2008) Independent protein-profiling studies show a decrease in apolipoprotein A1 levels in schizophrenia CSF, brain and peripheral tissues. Mol Psychiatry 13(12):1118-1128

31. Johnston-Wilson NL, Sims CD, Hofmann JP, Anderson L, Shore AD et al (2000) Disease-specific alterations in frontal cortex brain proteins in schizophrenia, bipolar disorder, and major depressive disorder. The Stanley Neuropathology Consortium. Mol Psychiatry 5:142-149

32. Khawaja X, Xu J, Liang JJ, Barrett JE (2004) Proteomic analysis of protein changes developing in rat hippocampus after chronic antidepressant treatment: implications for depressive disorders and future therapies. J Neurosci Res 75(4):451-460

33. Kim S, Choi KH, Baykiz AF, Gershenfeld HK (2007) Suicide candidate genes associated with bipolar disorder and schizophrenia: an exploratory gene expression profiling analysis of post-mortem prefrontal cortex. BMC Genom 8:413

34. Klempan TA, Sequeira A, Canetti L, Lalovic A, Ernst C et al (2009) Altered expression of genes involved in ATP biosynthesis and GABAergic neurotransmission in the ventral prefrontal cortex of suicides with and without major depression. Mol Psychiatry $14(2): 175-189$

35. Krömer SA, Kessler MS, Milfay D, Birg IN, Bunck M et al (2005) Identification of glyoxalase-I as a protein marker in a mouse model of extremes in trait anxiety. J Neurosci 25(17):4375-4384

36. Kubicki M, Park H, Westin CF, Nestor PG, Mulkern RV et al (2005) DTI and MTR abnormalities in schizophrenia: analysis of white matter integrity. Neuroimage 15:1109-1118

37. Kulkarni VA, Jha S, Vaidya VA (2002) Depletion of norepinephrine decreases the proliferation, but does not influence the survival and differentiation, of granule cell progenitors in the adult rat hippocampus. Eur J Neurosci 16:2008-2012

38. Lalovic A, Levy E, Canetti L, Sequeira A, Montoudis A, Turecki G (2007) Fatty acid composition in postmortem brains of people who completed suicide. J Psychiatry Neurosci 32(5):363-370

39. Lalovic A, Levy E, Luheshi G, Canetti L, Grenier E et al (2007) Cholesterol content in brains of suicide completers. Int $\mathbf{J}$ Neuropsychopharmacol 10(2):159-166

40. Lalovic A, Klempan T, Sequeira A, Luheshi G, Turecki G (2009) Altered expression of lipid metabolism and immune response genes in the frontal cortex of suicide completers. J Affect Disord [Epub ahead of print]

41. Lee J, Duan W, Mattson MP (2002) Evidence that brain derived neurotrophic factor is required for basal neurogenesis and mediates, in part, the enhancement of neurogenesis by dietary restriction in the hippocampus during contextual learning. J Neurochem 82:1367-1375

42. Lee SP, Ataga KI, Zayed M, Manganello JM, Orringer EP et al (2007) Phase I study of eptifibatide in patients with sickle cell anaemia. Br J Haematol 139(4):612-620 
43. Levin Y, Wang L, Schwarz E, Koethe D, Leweke FM, Bahn S (2009) Global proteomic profiling reveals altered proteomic signature in schizophrenia serum. Mol Psychiatry [Epub ahead of print]

44. Link AJ, Eng J, Schieltz DM, Carmack E, Mize GJ et al (1999) Direct analysis of protein complexes using mass spectrometry. Nat Biotechnol 17(7):676-682

45. Malberg JE, Eisch AJ, Nestler EJ, Duman RS (2000) Chronic antidepressant treatment increases neurogenesis in adult rat hippocampus. J Neurosci 20:9104-9110

46. Martins-de-Souza D, Gattaz WF, Schmitt A, Maccarrone G, Hunyadi-Gulyás E et al (2009) Proteomic analysis of dorsolateral prefrontal cortex indicates the involvement of cytoskeleton, oligodendrocyte, energy metabolism and new potential markers in schizophrenia. J Psychiatr Res 43:978-986

47. Martins-de-Souza D, Gattaz WF, Schmitt A, Rewerts C, Marangoni $S$ et al (2009) Alterations in oligodendrocyte proteins, calcium homeostasis and new potential markers in schizophrenia anterior temporal lobe are revealed by shotgun proteome analysis. J Neural Transm 116:275-289

48. Martins-de-Souza D, Gattaz WF, Schmitt A, Rewerts C, Maccarrone $G$ et al (2009) Prefrontal cortex shotgun proteome analysis reveals altered calcium homeostasis and immune system imbalance in schizophrenia. Eur Arch Psychiatry Clin Neurosci 259:151-163

49. Martins-de-Souza D, Gattaz WF, Schmitt A, Novello JC, Marangoni $S$ et al (2009) Proteome analysis of schizophrenia patients Wernicke's area reveals an energy metabolism dysregulation. BMC Psychiatry 9:17

50. Martins-de-Souza D (2009) Proteomics is not only a biomarker discovery tool. Proteomics Clin Appl 3:1136-1139

51. Martins-de-Souza D, Maccarrone G, Wobrock T, Zerr I, Gormanns P et al (2010) Proteome analysis of the thalamus and cerebrospinal fluid reveals glycolysis dysfunction and potential biomarkers for schizophrenia. J Neurosci (submitted)

52. Martins-de-Souza D, Dias-Neto E, Schmitt A, Falkai P, Gormanns P et al (2010) Proteome analysis of schizophrenia brain tissue. World J Biol Psychiatry (in press)

53. McKinnon MC, Yucel K, Nazarov A, MacQueen GM (2009) A meta-analysis examining clinical predictors of hippocampal volume in patients with major depressive disorder. J Psychiatry Neurosci 34(1):41-54

54. McLoughlin GA, Ma D, Tsang TM, Jones DN, Cilia J et al (2009) Analyzing the effects of psychotropic drugs on metabolite profiles in rat brain using $1 \mathrm{H}$ NMR spectroscopy. J Proteome Res 8(4):1943-1952

55. Morcuende S, Gadd CA, Peters M, Moss A, Harris EA et al (2003) Increased neurogenesis and brain-derived neurotrophic factor in neurokinin-1 receptor gene knockout mice. Eur J Neurosci 18(7): 1828-1836

56. Mu J, Xie P, Yang ZS, Yang DL, Lv FJ et al (2007) Neurogenesis and major depression: implications from proteomic analyses of hippocampal proteins in a rat depression model. Neurosci Lett 416(3):252-256

57. Nibuya M, Morinobu S, Duman RS (1995) Regulation of BDNF and trkB mRNA in rat brain by chronic electroconvulsive seizure and antidepressant drug treatments. J Neurosci 15:7539-7547

58. Novikova SI, He F, Cutrufello NJ, Lidow MS (2006) Identification of protein biomarkers for schizophrenia and bipolar disorder in the postmortem prefrontal cortex using SELDI-TOF-MS ProteinChip profiling combined with MALDI-TOF-PSD-MS analysis. Neurobiol Dis 23(1):61-76

59. Pennington K, Dicker P, Dunn MJ, Cotter DR (2008) Proteomic analysis reveals protein changes within layer 2 of the insular cortex in schizophrenia. Proteomics 8:5097-5107
60. Prabakaran S, Swatton JE, Ryan MM, Huffaker SJ, Huang JT et al (2004) Mitochondrial dysfunction in schizophrenia: evidence for compromised brain metabolism and oxidative stress. Mol Psychiatry 9:684-697 643

61. Prabakaran S, Wengenroth M, Lockstone HE, Lilley K, Leweke FM, Bahn S (2007) 2-D DIGE analysis of liver and red blood cells provides further evidence for oxidative stress in schizophrenia. J Proteome Res 6:141-149

62. Rosenzweig-Lipson S et al (2007) Differentiating antidepressants of the future: efficacy and safety. Pharmacol Ther 113:134-153

63. Salim K, Kehoe L, Minkoff MS, Bilsland JG, Munoz-Sanjuan I, Guest PC (2006) Identification of differentiating neural progenitor cell markers using shotgun isobaric tagging mass spectrometry. Stem Cells Dev 15(3):461-470

64. Sequeira A, Klempan T, Canetti L, ffrench-Mullen J, Benkelfat C et al (2007) Patterns of gene expression in the limbic system of suicides with and without major depression. Mol Psychiatry 12(7):640-655

65. Sequeira A, Mamdani F, Ernst C, Vawter MP, Bunney WE et al (2009) Global brain gene expression analysis links glutamatergic and GABAergic alterations to suicide and major depression. PLoS One 4(8): 6585

66. Sheline YI, Wang PW, Gado MH, Csernansky JG, Vannier MW (1996) Hippocampal atrophy in recurrent major depression. Proc Natl Acad Sci USA 93:3908-3913

67. Shirayama Y, Chen ACH, Nakagawa S, Russell DS, Duman RS (2002) Brain-derived neurotrophic factor produces antidepressant effects in behavioral models of depression. J Neurosci 22:32513261

68. Sibille E, Arango V, Galfalvy HC, Pavlidis P, Erraji-Benchekroun L et al (2004) Gene expression profiling of depression and suicide in human prefrontal cortex. Neuropsychopharmacology 29(2):351-361

69. Sivagnanasundaram S, Crossett B, Dedova I, Cordwell S, Matsumoto I (2007) Abnormal pathways in the genu of the corpus callosum in schizophrenia pathogenesis: a proteome study. Proteomics Clin Appl 1:1291-1305

70. Skolnick P, Popik P, Trullas R (2009) Glutamate-based antidepressants: 20 years on. Trends Pharmacol Sci [Epub ahead of print]

71. Skynner HA, Amos DP, Murray F, Salim K, Knowles MR et al (2006) Proteomic analysis identifies alterations in cellular morphology and cell death pathways in mouse brain after chronic corticosterone treatment. Brain Res 1102(1):12-26

72. Sultana R, Boyd-Kimball D, Cai J, Pierce WM, Klein JB et al (2007) Proteomics analysis of the Alzheimer's disease hippocampal proteome. J Alzheimers Dis 11(2):153-164

73. Tamminga CA, Thaker GK, Buchanan R, Kirkpatrick B, Alphs LD et al (1992) Limbic system abnormalities identified in schizophrenia using positron emission tomography with fluorodeoxyglucose and neocortical alterations with deficit syndrome. Arch Gen Psychiatry 49:522-530

74. Thambisetty M, Hye A, Foy C, Daly E, Glover A et al (2008) Proteome-based identification of plasma proteins associated with hippocampal metabolism in early Alzheimer's disease. J Neurol 255(11):1712-1720

75. Wilkins MR, Sanchez JC, Gooley AA, Appel RD, HumpherySmith I et al (1996) Progress with proteome projects: why all proteins expressed by a genome should be identified and how to do it. Biotechnol Genet Eng Rev 13:19-50

76. World Health Organization (2001) The World Health Report 2001: mental health: new understanding, new hope. World Health Organization, Geneva, p 169

77. Yao JK, Reddy RD, van Kammen DP (2001) Oxidative damage and schizophrenia: an overview of the evidence and its therapeutic implications. CNS Drugs 15:287-310 